\title{
Atención a la diversidad estudiantil en la virtualidad: desafíos docentes
}

\author{
Attention to student diversity in virtuality: teaching challenges
}

\author{
NOEMI AyQUIPA SALAZAR ${ }^{1}$ \\ noemi.ayquipa@unmsm.edu.pe \\ https://orcid.org/0000-0002-5875-1128 \\ Universidad Nacional Mayor de San Marcos, Perú
}

\section{RESUMEN:}

El presente artículo tiene como objetivo analizar el abordaje que se desplegó a la atención a la diversidad estudiantil en la virtualidad, durante la emergencia sanitaria por la Covid-19; además de resaltar los desafíos de la labor docente. El enfoque que ha seguido esta investigación ha sido cualitativo, de tipo básico; el método utilizado es la revisión sistemática de artículos y documentos que abordaron el tema. Durante la pandemia, los estudiantes con necesidades educativas especiales (NEE) han sido los más afectados, ante la falta de adaptaciones curriculares, recursos, materiales y plataformas virtuales que no han respondido a sus necesidades. Sin duda alguna, los docentes presentaban grandes desafíos en lo presencial; ahora, en la virtualidad, más aún. Tienen como reto adaptar todo el material, evaluar de manera individualizada a los estudiantes conociendo sus necesidades. Además, desde distintas esferas del Estado se debe vivir un enfoque inclusivo.

\begin{abstract}
:
The present article aims to analyze the approach that was deployed to the attention to student diversity in virtuality, during the health emergency caused by Covid-19; in addition to highlighting the challenges of teaching. The approach that this research has followed has been qualitative, of a basic type; the method used is the systematic review of articles and documents that dealt with the subject. During the pandemic, students with special educational needs (NEE) have been the most affected, due to the lack of curricular adaptations, resources, materials and virtual platforms that have not responded to their needs. Undoubtedly, the teachers presented great challenges in the classroom; now, in virtuality, even more so. Their challenge is to adapta II the material, to evaluate the students individually, knowing their needs. In addition, from different spheres of the State an inclusive approach must be lived.
\end{abstract}

\section{Palabras Clave:}

Atención a la diversidad; virtualidad; necesidades educativas especiales.

\section{KEYWORDS:}

Attention to diversity; virtuality; special educational needs. 


\section{Introducción}

Es la educación el instrumento más poderoso para transformar la vida de muchos hombres y mujeres del mundo. No obstante, según la UNESCO (2020) en uno de sus informes sobre situación de la educación a nivel mundial, se calcula que, alrededor de 258 millones de niños, niñas y jóvenes no tienen acceso a una educación básica y mucho menos de calidad. En este último punto, el Objetivo de Desarrollo Sostenible 4, planteado en la Agenda 2030, tiene como meta que todos los niños y niñas tengan una educación gratuita, equitativa e inclusiva (Blasco y Cid, 2020); sin embargo, es muy notoria la disparidad que existe en el mundo.

Antes de la pandemia, la educación enfrentaba grandes desafíos para lograr que esta sea inclusiva, con iguales oportunidades para todos. Estos problemas se agudizan frente la emergencia sanitaria que atravesamos por el Covid-19, donde los más afectados son los alumnos más vulnerables (alumnos con pobreza, pobreza extrema, con NEE, etc.) (Blasco y Cid, 2020). Ante ello, resalta el rol fundamental y relevante que desarrolla el docente para el logro de la tan ansiada inclusión educativa (García et al., 2018). Pero ante esta emergencia de salud por la Covid-19, se agudizan las dificultades con las que tiene que batallar el docente.

La educación virtual ha sido una alternativa para continuar con los aprendizajes de los estudiantes; sin embargo, la falta de un enfoque apropiado y de orientaciones oportunas para educar en la diversidad, por parte de las Instituciones Educativas, han limitado la participación, la permanencia y los aprendizajes de muchos estudiantes (Defensoría del pueblo, 2020). El informe especial número 36, expone que las familias peruanas, con hijos con necesidades educativas especiales (NEE), reconocen y señalan la falta de adaptaciones curriculares a las necesidades educativas, la ausencia de docentes especialistas en educación inclusiva; además, la carencia o falta de preparación del personal (docente, directivo, etc.) para atender la diversidad en el aula (Defensoría del pueblo, 2020).

Hay la necesidad de revisar y analizar cuáles son las competencias pedagógicas que debe tener o desarrollar un docente, para atender a esta diversidad en el aula. En el marco de buen desempeño docente (MBDD) se especifica cuatro dominios, en dos de ellos se abordan la preparación y la enseńanza para el aprendizaje de los estudiantes; el primero, cuenta con dos competencias que involucran el conocer, comprender y planificar experiencias de aprendizaje de teniendo en cuenta a las características de cada uno de los estudiantes; mientras que en el segundo dominio, las competencias están orientadas a crear un clima propicio donde los estudiantes se desarrollen con libertad a través de la convivencia armoniosa, donde abrace la diversidad (MINEDU, 2014).

Hablar de competencia no solo hace referencia a la facultad de solucionar un problema o alcanzar una meta, sino que implica todo una movilización de acciones internas y externas; es decir, conocimientos, actitudes, habilidades y destrezas (Marza y Cruz, 2018, como se citó en Lévano-Francia et al., 2019; Universidad Nacional de Educación, 2020); además, según uno de los instrumentos que rigen la política educativa del Estado peruano una competencia involucra "... un actuar reflexivo que a su vez implica una movilización de recursos tanto internos como externos, con el fin de generar respuestas pertinentes en situaciones problemáticas y la toma de decisiones en un marco ético" (MINEDU, 2014).

El MBDD es uno de los documentos relevantes que debe tener en cuenta un docente peruano, durante el desarrollo de su carrera profesional; ya que es una guía que permitirá alcanzar las metas u objetivos planteados con Estado. Según el MINEDU (2014), está comprendida por cuatro dominios de donde se desprenden nueve competencias docentes. En este estudio se ha analizado los dos primeros dominios que guardan relación con el aprendizaje de los estudiantes. En ese sentido, se hace mención de manera explícita a la atención a la diversidad o necesidades especiales en tres competencias y en cinco desempeños: competencia 1 (desempeño 1), competencia 3 (desempeńos 13, 14 y 16) y en la competencia 4 (desempeño 24); es decir, de los 29 desempeños que se presentan en las cinco primeras competencias, solo seis están referidas exclusivamente a la atención a estudiantes con NNE. 
La educación inclusiva es que todos tengan acceso a las mismas oportunidades educativas independientemente de su condición (Toquero, 2020). Es preciso saber que para que ello ocurra, hay distintas entidades u organismos que por ley deben actuar para garantizar la educación para todos. Es así que uno de los decretos legislativos dados en el 2020, hace responsables a distintos organismos e instancias como el Ministerio de educación (MINEDU), las Direcciones Regionales de Educación (DRE), las Unidades de Gestión Educativa Local (UGEL) y las distintas instituciones educativas (II.EE.), a velar por la continuidad educativa de todas las personas con NEE, proporcionándoles el acceso a distintos recursos educativos de acuerdos a sus características propias.

Bajo las resoluciones viceministeriales dadas por el MINEDU, en el año 2020, se hacen responsables a la DRE, UGEL y el mismo Ministerio de proporcionar, a los maestros, soporte sobre los contenidos y recursos educativos y así asegurar el desarrollo de la educación no presencial. Además, la Institución Educativa es la encargada de asegurar que todos los estudiantes continúen sus estudios, realizando gestiones para que todos los educandos tengan acceso a los recursos educativos.

Son distintas instituciones que, durante la emergencia sanitaria por la Covid-19, han tenido que unir sus esfuerzos para hacer que ninguno se quede atrás, es por eso que es de vital importancia saber qué competencias necesitan desarrollar los maestros para atender a la diversidad en el aula. Además, mirar cómo han respondido, a los niños con NEE, durante la pandemia.

De acuerdo a la investigación, las competencias emocionales, tales como la gestión del estrés, dinamismo y colaboración, autoconocimiento, actitud emprendedora, tolerancia, actitud pragmática y resolutiva, autorregulación, ética, integridad y valores humanos, comunicativo y relaciones personales son necesarias que se desarrollen y evalúen en los orientadores educativos, pues a través del trabajo colaborativo con toda la comunidad, podrá orientar de manera efectiva, más ajustada a la realidad y direccionar a una educación de calidad orientada a una inclusión verdaderamente efectiva (Fernández y Malvar, 2020).

Para lograr la tan ansiada educación inclusiva, no es suficiente tener una buena infraestructura y contar con todos los equipos necesarios para realizar el trabajo, sino que son relevantes las competencias docentes. En ese sentido, se propone en una educación universitaria que los docentes desarrollen competencias pedagógicasdidácticas, de aprendizaje cooperativo, de investigación, interactivas, éticas y competencias sociales; para que así pueda atender de manera efectiva y eficaz a la diversidad en el aula, y esto no solo se refiere a los estudiantes con discapacidad sino también a aquellos que tienen ritmos de aprendizaje distintos (Ayala y O’Hinggins, 2019). La inclusión no solo debe darse en las escuelas de educación básica sino también en la educación superior y universidades.

Para que los sistemas educativos sean verdaderamente inclusivos se debe contar con docentes inclusivos; es decir, que sean capaces de atender a la diversidad en el aula. Se propone que las competencias que debe poseer el maestro son la capacidad de enseñar, desarrollo del trabajo colaborativo con grupos de docentes, creación de espacios para el desarrollo emocional y social, aplicación de metodología y didáctica apropiadas. Además, se propone la estrategia pedagógica basada en competencias: aula invertida, que permitirá darles mayor protagonismo a los estudiantes, donde se pongan a prueba y movilice distintas capacidades. Con esta estrategia se propone que el estudiante asuma el rol del maestro, y pueda realizar trabajos de manera autónoma y colaborativa, aprendizaje basado en problemas y realización de proyectos (Montañez y Suárez, 2019).

Los estudios muestran que son aún escasas las investigaciones que se han realizado, en español, sobre la educación inclusiva; pese a ello, a lo largo de los últimos 25 años se han venido desarrollando cambios sustanciales en la políticas educativas y programas de formación docente. Sin embargo, sigue siendo relevante enfocarse en las cualidades y habilidades que debe tener el profesorado como conocimientos pedagógicos, didácticas especiales, aptitudes para actuar de manera inclusiva, ser buena persona, ser íntegro y ser educado. En este último punto, educar se relaciona con la capacidad de promover el desarrollo integral de la persona (Iglesias y Martín, 2020). 


\section{Metodología}

El objetivo del estudio fue analizar cómo se ha atendido a los estudiantes con NEE, en esta situación de pandemia por la Covid-19, además de reconocer cuales son las competencias docentes y comprender los desafíos que atraviesan los maestros, en esta virtualidad, para atender a la diversidad estudiantil. Esto se logró a través de la revisión de distintos documentos y artículos que abordaron el tema. Por tal razón, el estudio se basó en un enfoque cualitativo, el cual, según Hernández et al. (2018), nos indica que sigue un proceso flexible que nos permite retornar a cualquier etapa de la investigación para volver analizarla o interpretarla. El tipo de investigación que se desarrolló es básico, pues se centra en conocer y comprender el problema, a través de la obtención y recopilación de información, más no en la aplicación práctica (Sánchez y Reyes, 2015).

La investigación ha seguido un diseño fenomenológico, ya que a se ha buscado entender las experiencias de las familias con hijos con NEE, e interpretarlas (Hernández et al., 2018). Posteriormente, para la obtención de información se ha hecho uso del análisis documental, que permitió recabar datos y experiencias importantísimas para el enriquecimiento del marco teórico (Sánchez y Reyes, 2015). Para el recojo de información, se seleccionó y analizó datos de diversas fuentes bibliográficas, donde se hizo uso del gestor de referencias: Mendeley, que automatizó la recolección de datos y su posterior referenciación. Se tuvo en cuenta tres criterios, para selección de documentos: temporalidad, no mayor a 5 años; fuentes nacionales e internacionales que hayan abordado el tema; y por último, la definición de conceptos claves como inclusión educativa, diversidad en el aula y competencia docente.

\section{Resultados}

Se presentan los resultados de acuerdo al análisis documental, de tres relevantes documentos: el primero, corresponde al informe especial elaborado por la Defensoría del Pueblo, como respuesta a todo un análisis de las acciones que se habían desplegado "a favor" de los estudiantes con NNE en el Perú; el segundo, analiza las recomendaciones que da la UNICEF (2020) a todos los países a nivel mundial, sobre las consideraciones que deben tener las personas con discapacidad o necesidades especiales; y por último, el Marco de Buen Desempeño Docente.

\section{Resultados según la serie de informe especial de la Defensoría del Pueblo.}

- Acerca de los responsables de la continuidad de la educación inclusiva durante la pandemia.

Cuando hace mención a la disponibilidad, en el documento, esta consiste en garantizar los servicios integrales, idóneos y suficientes al ofrecer la prestación del servicio educativo. Ante la emergencia sanitaria, el Estado peruano dispuso, a través del decreto legislativo No 1468, acciones de prevención y protección para las personas con discapacidad. Específicamente en el artículo 4.6, se hace referencia a la continuidad del sistema educativo para personas con necesidad educativas especiales (NEE), donde se hacen responsables al MINEDU, las DRE), las UGEL e II.EE. de la incorporación de un enfoque de discapacidad en el sistema educacional de los distintos niveles: Educación Básica Regular (EBR), Educación Básica Alternativa (EBA), Educación Básica Especial (EBE) y Educación Técnica Productiva (ETP), donde se procure el acceso a distintos recursos educativos de acuerdo a las características propias de dicha población estudiantil. Sin embargo, tal tarea no se ha cumplido a cabalidad.

Una de las más grandes dificultades que han tenido que enfrentar, los niños y niñas con NEE y discapacidad, han sido la falta de ajustes o restructuración de materiales educativos y la falta de accesibilidad a las plataformas virtuales de aprendizaje, la cual tenía como responsables a las entidades mencionadas en el párrafo anterior.

Los años comprendidos entre el 2019-2020, hubo 880174 estudiantes con NEE con discapacidad matriculados; de los cuales el $70,32 \%$ pertenecieron a la EBR, nuestro sistema educativo no es 
inclusivo muy por el contrario es excluyente, y ello parte desde la entidad más alta e importante: el MINEDU, pues las normas técnicas dadas no siguen un enfoque de inclusión, inclusive desde antes de la pandemia. Ello se hace mucho más evidente en tiempos de pandemia; pues se observan en las clases del programa "Aprendo en casa" que durante tres semanas de transmisión no se pensó en las niñas, niños y jóvenes sordos. Ninguno de sus programas de televisión, transmitidos en ese lapso de tiempo, usaron intérpretes de Lengua de Señas Peruana (LSP), ni adaptaron un solo recurso para las personas con discapacidad intelectual leve o moderada, discapacidad auditiva, visual, física, con Trastorno de Espectro Autista (TEA), TDAH, diferente estilo de aprendizaje o enfermedades raras. La respuesta después de esas semanas, fue la aparición del intérprete, pero un pequeño recuadro, que no permitía discriminar con claridad las señas.

Los contenidos que se comparten en el programa "Aprendo en casa", para la población estudiantil NEE están orientadas a los padres de familia más no a los estudiantes, y solo se transmite vía radio. La defensoría del pueblo ha recomendado colocar subtítulos a las sesiones transmitidas en televisión, ya que no todos los estudiantes con discapacidad auditiva se comunican mediante señas. Además, como medida de apoyo a las instituciones de educación básica especial se le ha creado el SAANEE Central (Servicio de Apoyo y Asesoramiento para la Atención de Necesidades Educativas Especiales Central), el cual atiende a docentes y no docentes brindándoles apoyo técnico virtual en cuanto a las adaptaciones curriculares y pedagógicas.

\section{- Acerca de las impresiones de los padres de familia.}

Se recabó información sobre la percepción que tenían los padres de familias a cerca del servicio educativo que recibían sus hijos con NEE y discapacidad. Se realizaron encuestas virtuales a un total de 50 familias, cuyos hijos estaban en escuelas públicas y privadas. Muchas de ellas manifestaron su disconformidad con los servicios educativos prestados, pues no se consideraba, en muchos casos, a sus hijos pues no se adaptaban los materiales y contenidos a sus necesidades; además, indicaban que se evidenciaba la falta de preparación del personal de las II.EE. para atender a la diversidad estudiantil, no se contaban con docentes especialistas en educación inclusiva que puedan dar soporte.

Parafraseando las palabras de algunos padres, con hijos con discapacidad intelectual y con TDAH, mencionan que la mayor parte del tiempo de clases está orientado a un estudiante sin discapacidad, que las plataformas empleadas no cuenta con materiales adaptados a las necesidades de sus hijos, no hay intervención ni orientación por parte del director ni de los docentes.

\section{Resultados de acuerdo a las consideraciones que da la UNICEF para personas con discapacidad.}

La UNIFEC recomendó proporcionar apoyo a los distintos actores educativos para que puedan garantizar el uso de distintas plataformas de aprendizajes a distancia que sean accesibles y seguras para los nińos con discapacidad. Además, sugieren brindar capacitación a los maestros para que puedan brindar soporte y apoyo a los niños en las clases remotas. Que cualquier programa que se plantee en la educación especial se incluyan las medidas necesarias que garanticen la continuidad de la educación para los niños, niñas, jóvenes y adultos.

\section{Resultados según el MBDD.}

- Acerca de los dominios, competencias y desempeños de los docentes, referidos a la atención a la diversidad en el aula.

El MBDD es un documento importantísimo que guía la labor del docente peruano, ya que busca establecer cuáles son las competencias por ende los desempeños que deben realizar los maestros. Este marco cuenta con cuatro dominios, de los cuales los dos primeros son los que se relacionan con la preparación y desarrollo de la enseñanza en el aula; además, en ambas se mencionan que siguen un 
enfoque intercultural e inclusivo que atiende a la diversidad en todas sus expresiones. Los dos dominios restantes, están orientados a la gestión escolar con las familias y el desarrollo profesional docente.

A continuación, se hace mención a las competencias y desempeños que se relacionan con la atención a la diversidad en las aulas, en la tabla siguiente:

Tabla 1

Dominios, competencias y desempeños

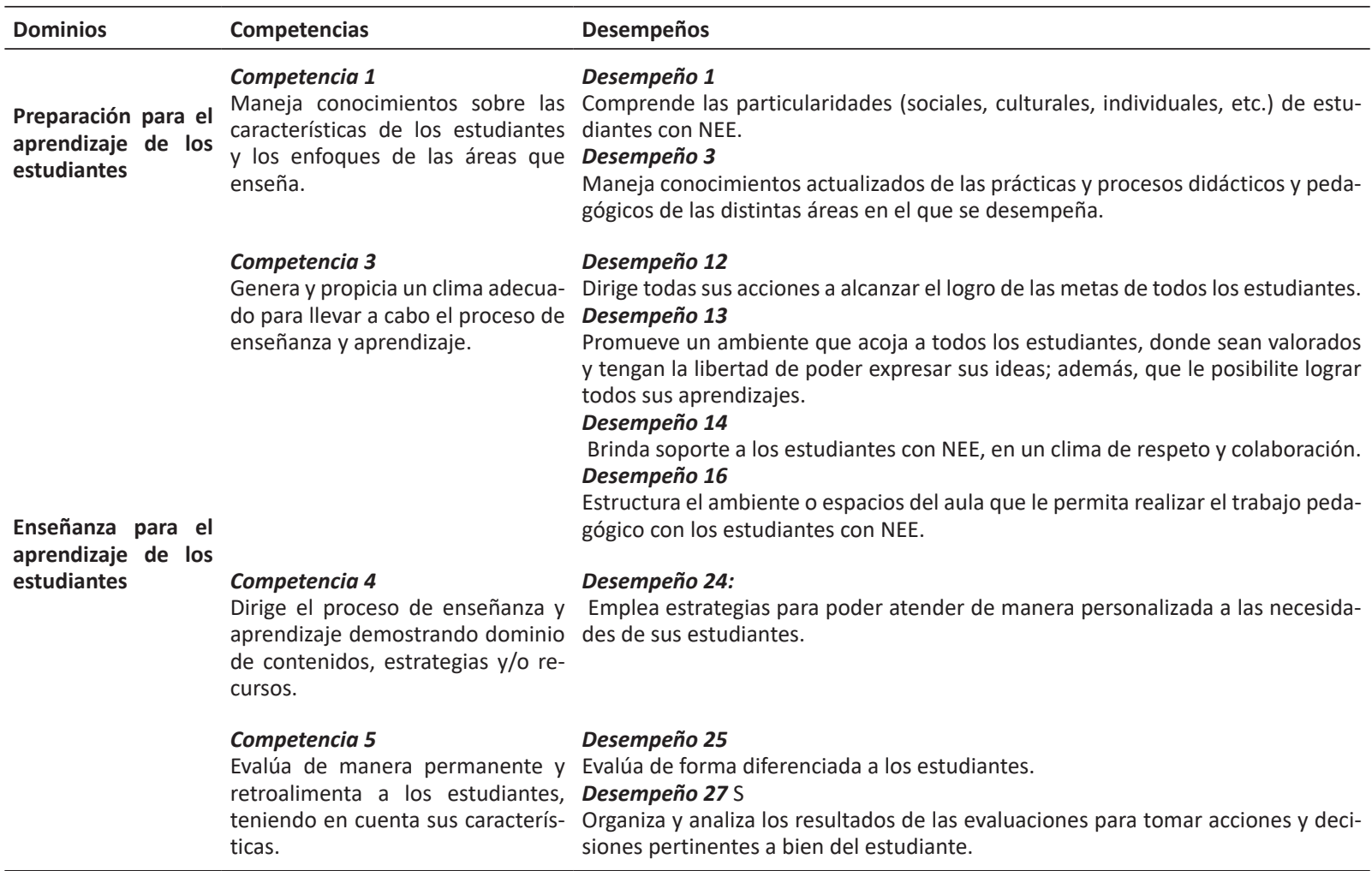

Fuente: MINEDU (2014)

\section{Discusión}

La presente investigación se planteó como objetivo, analizar la atención que se brindó durante la pandemia a los niños, niñas y jóvenes con NEE, reconocer las competencias docentes para atender a la diversidad estudiantil en el aula, además de identificar los desafíos que se les presentan a los maestros. El 15 de marzo de 2020, el Perú se declara en estado de emergencia nacional ante los casos por Covid-19, que iban en crecimiento (DS No 044-2020-PCM, 2020). En un primer momento, se piensa retornar a clases luego de 15 días; sin embargo, ante la curva de crecimiento se oficializa el inicio de clases virtuales el 6 de abril del mismo ańo, con la estrategia "Aprendo en casa" para escuelas públicas (RM No 160-2020-MINEDU, 2020), mientras que algunas instituciones privadas iban explorando y poniendo en práctica el uso de distintas plataformas e-Learning como Zoom y Meet.

Cuando la estrategia Aprendo en casa empezó a aplicarse, no se consideró a las personas con necesidades educativas especiales; pues en los programas transmitidos por internet y televisión no se consideraron a las personas sordas; además los contenidos no estaban adaptados para la diversidad que existía en las aulas (Defensoría del Pueblo, 2020). Lo planteado por el gobierno no es coherente con lo que proponen: garantizar la continuidad de la educación a las personas con discapacidad en los distintos niveles, modalidades y etapas en las que se desarrollan. Se ha observado y vivenciado que las distintas entidades, y por ende los maestros, no han sido empáticos con este grupo de estudiantes y sus familias. Según lo propuesto por Ayala y O’Hinggins (2019), hay una necesidad de docentes competentes con cultura inclusiva que sean capaces de generar o 
propiciar oportunidades para el estudiante al margen de sus condiciones educativas. Podríamos añadir que no solo los docentes deben desarrollar dicha cultura o enfoque inclusivo sino también todas las entidades del Estado, no solo en papel sino vivirlo.

Además, el Estado indica claramente que se tienen que realizar adaptaciones y facilitar el acceso de recursos educativos que puedan apoyar su educación (DL No 1468, 2020); sin embargo, esto no se cumplió y se puede constatar en el informe que realizó la Defensoría del Pueblo al cierre del año 2020. El MBDD en la competencia 4, desempeño 24 hace mención a que el maestro competente debe manejar distintas y estrategias pedagógicas y didácticas para así poder atender de manera personalizada a los estudiantes con NEE (MINEDU, 2014). Por lo tanto, podemos inferir que no todos los maestros están desarrollando estas competencias, pues aún presentan deficiencias al preparar un material o recursos pedagógicos que sean capaces de guiar el aprendizaje de los estudiantes y posteriormente, permitirles lograr las metas que establece el ministerio.

Muchas familias estuvieron inconformes con el servicio prestado tanto en las II.EE. públicas como privadas, pues no se realizaban las adaptaciones curriculares ni de recursos; los maestros no se encontraban capacitados para brindar apoyo y sostenerlos; las distintas instituciones no brindaban soporte a las familias para el trabajo en casa con sus hijos, dejando, a muchos de ellos, a la deriva (Defensoría del Pueblo, 2020). Sin duda alguna, los maestros han enfrentado un gran desafío al pasar de las clases presenciales a las clases virtuales; no obstante, se conoce que ellos deben haber desarrollado o estar desarrollando algunas competencias que lo establecen los documentos que dirigen su quehacer pedagógico. El MBDD dentro de las competencias 1, 3, 4 y 5 promueven la atención a la diversidad en el aula, se cuenta con nueve desempeños donde que miden si el maestro es o no competente en su labor docente (MINEDU, 2014).

Para Alaya y O’Higgins (2019) las competencias básicas, que deben dominar los maestros, son "conocimientos y emplear valores actitudinales hacia la inclusión, dominar las tecnologías de la información y la comunicación (TIC), aprender, aplicar y desarrollar pensamiento lógico-crítico, utilizar estrategias de enseñanza-aprendizaje, contar con destrezas curriculares transversales, fomentar la investigación y reflexión sobre las necesidades detectadas" (p.81). La investigación no solo debe darse en las universidades sino también deben ponerse en práctica en las escuelas, generando maestros investigadores, propositivos, que busquen soluciones a los desafíos que se presentan en la escuela. Las competencias sociales y emocionales son muy relevantes, en docentes que atienden a estudiantes con NEE, tales como la integridad, ética, autorregulación, tolerancia, trabajo en equipo, dinamismo, gestión del estrés, autoconocimiento y colaboración (Fernández y Malvar, 2020; Montañez y Suárez, 2019; Iglesias y Martín, 2020).

\section{Conclusiones}

Se puede concluir que (1) hay una necesidad de vivir un enfoque inclusivo no solo en los documentos sino desde las acciones que realizan las distintas instituciones del Estado, desde el Ministerio de Educación hasta las escuelas; además se debe seguir (2) empoderando y orientando al maestro a desarrollar las competencias establecidas en el MBDD; allí es muy importante la gestión del director porque es quien debe acompañar, guiar, orientar y retroalimentar la labor docente. (3) Hay una necesidad de incorporar competencias sociales y emocionales dentro del MBDD. (4) Las estrategias planteadas en "Aprendo a casa" deben ser nuevamente revisadas y replanteadas, que estén orientadas a atender las necesidades de estudiantes con NEE (DL No 1468, 2020; UNICEF, 2020).

\section{Referencias}

Ayala, S., y OHinggins, T. (2019). Perfil docente de la educación superior inclusiva en Paraguay Teaching profile of inclusive higher education in Paraguay. Revista Cientifica En Ciencias Sociales UP, 1(1), 75-84. http://www. upacifico.edu.py:8040/index.php/PublicacionesUP_Sociales/article/view/21 
Blasco, E. P., y Cid, M.S. (2020). La Agenda 2030. In Hablamos de derechos humanos. https://doi.org/10.2307/j. ctv153k4pm.7

Defensoría del pueblo. (2020). El derecho a la educación inclusiva en el contexto de la emergencia sanitaria por el Covid-19. https://www.defensoria.gob.pe/wp-content/uploads/2020/12/Serie-Informes-especiales-36_ Educaci\%C3\%B3n-inclusiva-en-contexto-de-COVID-19.pdf

D.L. No 1468-2020-Congreso de la República. Decreto Legislativo que establece disposiciones de prevención y protección para las personas con discapacidad ante la Emergencia Sanitaria ocasionada por la Covid-19. Diario Oficial El Peruano (2020). https://busquedas.elperuano.pe/normaslegales/decreto-legislativo-que-establecedisposiciones-de-prevencio-decreto-legislativo-n-1468-1865717-2/

D.S. No 044-2020-PCM. Que declara Estado de Emergencia Nacional por las graves circunstancias que afectan la vida de la Nación a consecuencia del brote del COVID-19. Diario Oficial El Peruano (2020). https://www.gob.pe/ institucion/pcm/normas-legales/460472-044-2020-pcm

Fernández, M. D., y Malvar, M. L. (2020). Las competencias emocionales de los orientadores escolares desde el paradigma de la educación inclusiva. Revista de Investigación Educativa, 38(1), 239-257. https://doi.org/10.6018/ rie.369281

García, C., Herrera, C., y Vanegas, C. (2018). Competencias docentes para una pedagogía inclusiva. Consideraciones a partir de la experiencia con formadores de profesores chilenos. Revista Latinoamericana de Educación Inclusiva, 12(2), 149-167. https://scielo.conicyt.cl/scielo.php?script=sci_abstract\&pid=S0718-73782018000200149\&lng=e s\&nrm=iso

Hernández-Sampieri, R. y Mendoza, T. C. (2018). Metodología de la Investigación. Las rutas cuantitativa, cualitativa y mixta. Mc Graw Hill Education

Iglesias, A. y Martín, Y. (2020). La producción científica en educación inclusiva: avances y desafíos. Revista Colombiana de Educación, 78, 383-418. http://doi.org/10.17227/rce.num78-9885

Levano-Francia, L., Sánchez, S. Guillen-Aparicio, P., Tello-Cabello, S., Herrera-Paico, N., y Collantes-Inga, Z. (2019). Competencias digitales y educación. Propósitos y Representaciones, 7(2), 569-588. http://dx.doi.org/10.20511/ pyr2019.v7n2.329

MINEDU. (2014). Para mejorar tu práctica como maestro y guiar el aprendizaje de tus estudiantes. http://www. minedu.gob.pe/pdf/ed/marco-de-buen-desempeno-docente.pdf

Montañez, T. C. y Suárez, Z. M., (2019). Artículo de investigación Conceptualización de la formación profesional para la educación inclusiva. Conceptualization of training profesional for inclusive education. Revista Electrónica de Investigación e Innovación Educativa. 1, 125-140. http://webcache.googleusercontent. com/search?q=cache:BWaePhuoOfAJ:cresur.edu.mx/OJS/index.php/CRESUR_REIIE/article/ view/320/238+\&cd=1\&hl=es\&ct=clnk\&gl=pe

R.M No 160-2020-MINEDU. (2020). Que disponen el inicio del año escolar a través de la implementación de la estrategia demoniada "Aprendo en casa", a partir del 6 de abril de 2020 y aprueban otras disposiciones. Diario Oficial El Peruano (2020). https://www.gob.pe/institucion/minedu/normas-legales/466108-160-2020-minedu

Sánchez, H. y Reyes, C. (2015). Metodología y Diseños en la Investigación Científica. Business Support Aneth.

Toquero, C. M. (2020). Challenges and Opportunities for Higher Education amid the COVID-19 Pandemic: The Philippine Context. Pedagogical Research, 5(4), em0063. https://doi.org/10.29333/pr/7947

UNESCO. (2020). Inclusión y educación. In Educación Inclusiva. https://doi.org/10.2307/j.ctv153k3m3.6

UNICEF. (2020). Recomendaciones Covid-19: Consideraciones para niñas, niños y adultos con discapacidades. https://sites. unicef.org/disabilities/files/RESPUESTA_COVID_-_SPANISH.pdf

Universidad Nacional de Educación Enrique Guzmán y Valle Fondo Editorial. (2020). Walther Peñaloza Ramella. Vigencia de su enfoque educativo. Breve antología. http://www.une.edu.pe/uneweb/wp-content/uploads/2021/04/ Libro-Walter-Pe\%C3\%B1aloza-Ramella.pdf 


\section{Notas al final}

1 Docente de formación con especialización en educación primaria y con maestría en la mención gestión educativa por la Universidad Nacional Mayor de San Marcos del Perú. Ejerció la docencia de diferentes instituciones educativas públicas y privadas. Actualmente es docente de primaria de la institución educativa privada Saint Gerard School. 